\title{
El papel del sexo en comprensión lectora. Evidencias desde PISA y PIRLS
}

\section{The role of sex in reading comprehension. Evidence from PISA and PIRLS}

\author{
Silvia Fuentes de Frutos ${ }^{1}$, Víctor Renobell Santaren ${ }^{2}$ \\ ${ }^{1}$ Universidad Internacional de la Rioja. silvia.fuentes@unir.net \\ ${ }^{2}$ Universidad Internacional de la Rioja. victor.renobell@unir.net
}

Recibido: 6/4/2020

Aceptado: 16/10/2020

\section{Copyright (C)}

Facultad de CC. de la Educación y Deporte. Universidad de Vigo

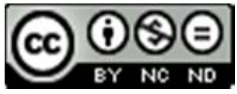

Dirección de contacto:

Víctor Renobell Santaren

Facultad de Educacion y Ciencias Sociales

Universidad Internacional de La Rioja

Av. de la Paz, 137

26006 Logroño, La Rioja

\begin{abstract}
Resumen
Existe evidencia empírica sobre la influencia del sexo en el proceso de adquisición y consolidación de la comprensión lectora a nivel internacional. El presente trabajo analiza si dicho fenómeno se reproduce también en el caso español. Para ello, se valora la existencia y evolución de una brecha de género en el rendimiento en comprensión lectora en España, basándose en los resultados de todas las ediciones PISA y PIRLS en las que España ha participado. Se examina también el rendimiento en comprensión lectora del alumnado español en dichas evaluaciones. La metodología mixta utilizada en el presente estudio se basa en una revisión bibliográfica de los últimos 25 años y en un análisis multisectorial de los datos proporcionados por los informes PISA y PIRLS. Los resultados de nuestro trabajo indican una mejor comprensión lectora en alumnas que en alumnos observable en todas las ediciones de PISA y PIRLS. Según la evolución de los resultados en PISA, la brecha de género se habría reducido en las últimas ediciones, algo no corroborado por PIRLS. Por otra parte, ambas evaluaciones relatan una mejora en el resultado global del alumnado español en comprensión lectora en las últimas ediciones.
\end{abstract}

\author{
Palabras clave \\ Evaluación Educativa, Comprensión Lectora, Género, PISA, PIRLS
}

\begin{abstract}
There is empirical evidence on the influence of sex in the process of acquisition and consolidation of reading comprehension at international level. This paper analyzes whether this phenomenon is also reproduced in the Spanish case. To this end, the existence and evolution of a gender gap in reading comprehension performance in Spain is assessed, based on the results of all the PISA and PIRLS editions in which Spain has participated. Reading comprehension performance of Spanish students is also examined. The mixed methodology used in this study is based on a literature review of the last 25 years and a multisectoral analysis of the data provided by the
\end{abstract}


PISA and PIRLS reports. Results of our work indicate better reading comprehension in girls than in boys observable in all editions of PISA and PIRLS. According to the evolution of PISA results, the observed gender gap would be reduced in recent editions, something not corroborated by PIRLS. On the other hand, both evaluations report an improvement in the overall result of Spanish students in reading comprehension in recent editions.

\section{Key Words}

Educational Evaluation, Reading Comprehension, Gender, PISA, PIRLS

\section{INTRODUCCIÓN}

El objetivo del presente estudio es valorar si existen diferencias en comprensión lectora en función del sexo en alumnos españoles de Educación Primaria (10 años) y Educación Secundaria (15 años). Además, se valorará también la evaluación del alumnado español (sin distinción de sexo) en comprensión lectora en las mismas etapas. Para ello se recurre al análisis de los resultados españoles en lectura en dos pruebas internacionales: PISA (Programme for International Student Assessment) y PILRS (Progress in International Reading Literacy Study).

Este estudio se basa en el uso de una metodología mixta. Se diferencian en él dos partes que se detallan a continuación. En primer lugar, se recurre a una revisión bibliográfica de los últimos 25 años, a partir de varias bases de datos (WOS, SCOPUS, Google Académico y Dialnet). Basándose en la misma, se presentan los factores influyentes más estudiados en relación con la comprensión lectora, haciendo especial hincapié en el sexo, cuestión central en nuestro trabajo. A continuación, se recurre al análisis multisectorial de los resultados de todo el alumnado español en PISA y PIRLS. Los datos que se analizan han sido facilitados por PISA, PIRLS y OCDE (Organización para la Cooperación y el Desarrollo Económicos).

Leer y comprender bien lo que se ha leído forma parte de la base educativa más esencial y necesaria. La correcta comprensión de un texto favorece todo proceso de aprendizaje en cualquier contexto educativo y supone un medio de acceso al nivel máximo de expresión simbólica. La comprensión lectora implica, del mismo modo, la adquisición de habilidades metalingüísticas, al generar esta consciencia sobre los fenómenos lingüísticos (Bohórquez, Cabal y Quijano, 2014).

Se han descrito diferencias en comprensión lectora en función de determinadas características del entorno de la persona. Los condicionantes familiares, sociales y económicos han demostrado ser influyentes en el nivel de comprensión lectora del alumnado (MECD, 2017). El tipo de experiencias previas a la escolarización, proporcionadas por el contexto familiar, puede llegar a facilitar considerablemente el rendimiento en comprensión lectora (Martín, 2010). El alumnado que vive en hogares con un mayor número de libros tiende a mostrar un mejor nivel en comprensión lectora y a mostrar un mayor nivel de resiliencia en lectura (MECD, 2017). Otra variable a la que se recurre de forma amplia para explicar las diferencias observadas es la titularidad del centro educativo. En dicho sentido, el alumnado que acude a centros privados presenta un mejor rendimiento en comprensión lectora que el alumnado que acude a centros públicos (MECD, 2013; MECD, 2017). 
En referencia a las características individuales, destaca el papel de la inteligencia como una variable con una importante incidencia en la comprensión lectora (BlázquezGarcés, Fernández, Sanz, Tijeras, Vélez y Pastor, 2015). Se ha descrito una correlación positiva entre un nivel alto de inteligencia y un buen nivel de comprensión lectora (Bohórquez et al, 2014; León, Amaya y Orozco, 2012). Se ha apuntado también hacia la existencia de una relación estrecha entre el rendimiento escolar en las diferentes materias (no solamente las lenguas) y la habilidad lectora (Blázquez-Garcés et al, 2015; Elosúa, García-Madruga, Gómez-Veiga, López-Escribano, Pérez y Orjales, 2012; Moje, Stockdill, Kim y Kim, 2011). Como ejemplo, se ha señalado que la relación entre comprensión lectora y ciencias es más fuerte que la existente entre comprensión lectora y matemáticas (MECD, 2017).

El propio interés y facilidad por la lectura, por parte del alumnado, parecen facilitar también el rendimiento en comprensión lectora. Cuanto mayor es el interés relatado de del alumnado por la lectura, mejor es su rendimiento en compresión lectora. Del mismo modo, el poseer un autoconcepto lector elevado mejora la ejecución en comprensión lectora (MEC, 2007; MECD, 2017). La confianza en la lectura se muestra como una variable que coadyuva a la resiliencia lectora (MECD, 2017).

Por último, es de especial interés hacer referencia al sexo. El sexo es una variable que se contempla de manera recurrente en relación con el rendimiento en comprensión lectora. Se debe señalar que son varios los estudios y trabajos internacionales que indican una mejor competencia lectora en alumnas, en comparación con los alumnos, tanto en Educación Primaria (Torres y Granados, 2014; Mullis, Martin, González y Kennedy, 2003), como en Educación Secundaria y pre-universitaria (Corpas, 2013; Julià, 2016), incluso en Lengua Extranjera (Corpas, 2013). Del mismo modo, las diferentes evaluaciones internacionales (PISA y PIRLS) describen ventaja de las alumnas en comprensión lectora (Julià, 2016).

Tal y como se ha apuntado, se trataría de una tendencia que se inicia desde los primeros años de aprendizaje, según describen algunos estudios (American Psychiatric Association, 2000; Roselli, Matute y Ardila, 2006). Durante la fase de aprendizaje de la lectura, se ha descrito que las alumnas superan a los alumnos en el ejercicio de pruebas basadas en la precisión y velocidad al leer y en saber distinguir los sonidos y las letras que forman las palabras (Roselli et al, 2006). Además de en comprensión lectora, la ventaja de las alumnas en habilidad verbal está referenciada internacionalmente (Mullis et al, 2003), lo mismo ocurre con la ventaja en habilidad para escribir (Bae, Choy, Geddes, Sable y Snyder, 2000).

Se ha subrayado la importancia de la atención, como aspecto cognitivo, y de la motivación, la persistencia en la tarea, la autonomía, la disciplina, la auto-eficacia o las habilidades organizativas, como aspectos no-cognitivos, como factores que ayudan a explicar las diferencias detectadas entre sexos en determinados trabajos. En los diferentes factores aquí mencionados, las alumnas presentan mejores puntuaciones (Duckworth y Seligman, 2006; Jacob, 2002; Julià, 2016). Para profundizar en la compresión de estos datos, resulta interesante el trabajo de Heckman, Stixrud y Urzua (2006), en el que se cuantifica el valor relativo de las habilidades cognitivas y las nocognitivas en el desarrollo. Algunos de los hallazgos de este trabajo indican que poseer un buen nivel de habilidades no-cognitivas a edades tempranas aumenta la probabilidad de tener un mayor desarrollo personal y social. 
Por otra parte, se ha demostrado que las alumnas disfrutan más de la lectura que los alumnos y que acuden a la misma como fuente de placer (MEFP, 2019), eligiendo temas distintos a los elegidos por los alumnos (McGeown, 2012). Existe una importante relación entre el rendimiento académico del alumnado y el disfrute de la lectura. Según se ha indicado, disfrutar de la lectura es requerimiento para alcanzar una lectura eficaz, lo que comporta una mejora de la competencia lectora (Mol y Jolles, 2014; OECD, 2015). Las alumnas también muestran un mayor interés por la escuela y los estudios que los alumnos (Jacob, 2002; Rosenbaum, 2001).

Los alumnos, en comparación con las alumnas, se muestran menos persistentes en las tareas académicas y son menos disciplinados que las alumnas (Jacob, 2002; Skiba et al, 2002). Además, aquellos suelen mostrar peores competencias sociales y comunicativas. Se ha demostrado que poseen más posibilidades de presentar comportamientos antisociales (Moffitt, 2001), de ser castigados (Cooley, 1995; Gregory, 1996), y de repetir curso que las alumnas (Freeman, 2004). Tal y como se ha constatado, todo ello estaría relacionado con el hecho de poseer menor habilidad lectora (Trzesniewski, Moffitt, Caspi, Taylor y Maughan, 2006).

Resulta interesante destacar que, en general, hasta la segunda mitad de los años 90, toda problemática en relación con el rendimiento educativo y el sexo se centraba en el análisis de las problemáticas de las alumnas solamente. A partir de ese momento, se comienzan a problematizar también los resultados educativos en alumnos y alumnas (Goldin, Katz y Kuziemko, 2006).

Para explicar las diferencias observadas en algunos trabajos en comprensión lectora, en función del sexo, se ha aludido a los efectos del tipo de crianza y del ambiente social, a los itinerarios escolares, al contexto social y cultural (que podría, por ejemplo, propiciar el logro educativo de un género más que del otro), a las prácticas y políticas educativas, y a las actitudes del alumnado con respecto a diferentes áreas. De esta manera las alumnas presentarían mayor compromiso frente a la lectura que los alumnos, pero mayor ansiedad que ellos frente a las matemáticas (OCDE, 2002, 2004, 2008, 2010). Todos estos aspectos estarían relacionados con la cultura de género y la transmisión de estereotipos, de manera continua, a través de los diferentes agentes socializadores.

Según Weiner (2010), históricamente se ha esperado que hombres y mujeres desempeñaran diferentes roles en la sociedad, cediendo el protagonismo a los hombres en la esfera pública y limitando a las mujeres al dominio privado. La adscripción de género ha colocado históricamente a hombres y mujeres en diferentes posiciones de poder. Esta perspectiva se ha seguido manteniendo hasta la actualidad. Se considera que los hombres son mejores en habilidades espaciales, numéricas y mecánicas y que las mujeres desarrollan habilidades verbales (saber escuchar, hablar, leer y escribir) de forma más competente y de manera más precoz. Además, las mujeres estarían más motivadas intrínsecamente por la lectura que los hombres (McGeown, Goodwin, Henderson y Wright, 2011).

Esta perspectiva conservadora sobre las diferencias de sexo influye en la educación, ya que se considera un medio para socializar y educar al alumnado en sus roles "naturales" como hombres y mujeres. Algunos trabajos etnográficos subrayan que la escuela posee un papel determinante en la generación de las identidades según el sexo y cómo esas identidades influyen en las actitudes y comportamientos hacia la educación (Francis, 2000; Skelton, 1997). 
Cabe destacar que, sin embargo, no todo el cuerpo de conocimiento relativo a la cuestión apunta hacia dicha dirección. Existe una serie de estudios y trabajos que niega la existencia de diferencias en función del sexo en comprensión lectora, tanto en Educación Primaria (Blázquez-Garcés et al, 2015), como en Educación Secundaria y pre-universitaria (Koban, 2016; Yazdanpanah, 2007). También se ha descrito que la percepción de la auto-eficacia lectora en Educación Primaria no depende del sexo (Olivares, Fidalgo y Torrance, 2016). Por otra parte, hay trabajos que refieren ventajas por parte de los alumnos, en comparación con las alumnas, en comprensión oral (Velarde, Canales y Meléndez, 2013).

\section{RESULTADOS}

El informe PISA (Programme for International Student Assessment) es un estudio de la OCDE (Organización para la Cooperación y el Desarrollo Económicos) que mide los conocimientos y destrezas del alumnado de 15 años en Lectura, Matemáticas y Ciencias. Esta evaluación internacional se realiza cada 3 años y es la encuesta educativa más amplia a día de hoy. En su edición más reciente, en 2018, participaron 79 países. El indicador que suele recibir más atención es el lugar ocupado por cada país según la puntuación media. España participa en esta evaluación desde el año de inicio, el 2000.

Por su parte, el estudio PIRLS (Progress in International Reading Literacy Study), es una evaluación de la IEA (Asociación Internacional para la Evaluación del Logro Educativo) que pone a prueba la comprensión lectora del alumnado en $4^{\circ}$ de Primaria cada cinco años desde el 2001. Se trata de una evaluación que se centra de manera exclusiva, a diferencia de PISA, en la comprensión lectora. PIRLS proporciona también información sobre el apoyo en el hogar y el ambiente escolar durante el proceso de aprendizaje de la lectura. España participa en este estudio desde la edición del 2006.

\begin{tabular}{|c|c|c|c|c|c|c|c|}
\hline PISA & 2000 & 2003 & 2006 & & 2009 & 2012 & 2015 \\
\hline España & 493 & 481 & 461 & & 481 & 488 & 495 \\
\hline Alumnas & 505 & 500 & - & * & 496 & 503 & 506 \\
\hline Alumnos & 481 & 461 & - & $*$ & 467 & 474 & 485 \\
\hline $\begin{array}{l}\text { Diferencia neta entre } \\
\text { alumnas y alumnos (en } \\
\text { puntos) }\end{array}$ & 24 & 39 & 35 & & 29 & 29 & 21 \\
\hline
\end{tabular}

Tabla 1. Media de resultados de PISA en España y por sexo.

*Los datos referentes al 2006 no constan en el informe de la OCDE.

La diferencia observada en las diferentes ediciones resulta significativa $(p=0.005)$

Fuente: Ministerio de Educación, Cultura y Deporte y OCDE

\subsection{Resultados PISA (Programme for International Student Assessment)}

\section{PISA 2000}

En PISA 2000 participaron 32 países, entre ellos España. La lectura fue el principal objeto de evaluación en esta edición. Se valoró la competencia lectora a través de tres subescalas diferentes: recuperación de información, interpretación de lo leído, y capacidad de reflexión sobre lo leído. Los resultados generales, sin distinción de sexo, 
muestran que el alumnado español tuvo mayor problema en recuperar la información (483 puntos, posición número 20 en la clasificación) que para interpretar la información (491 puntos, posición número 19 en la clasificación) o reflexionar sobre la misma (506 puntos, posición número 13 en la clasificación). En global, los resultados del alumnado español en lectura se acercan a la media de la OCDE (500 puntos).

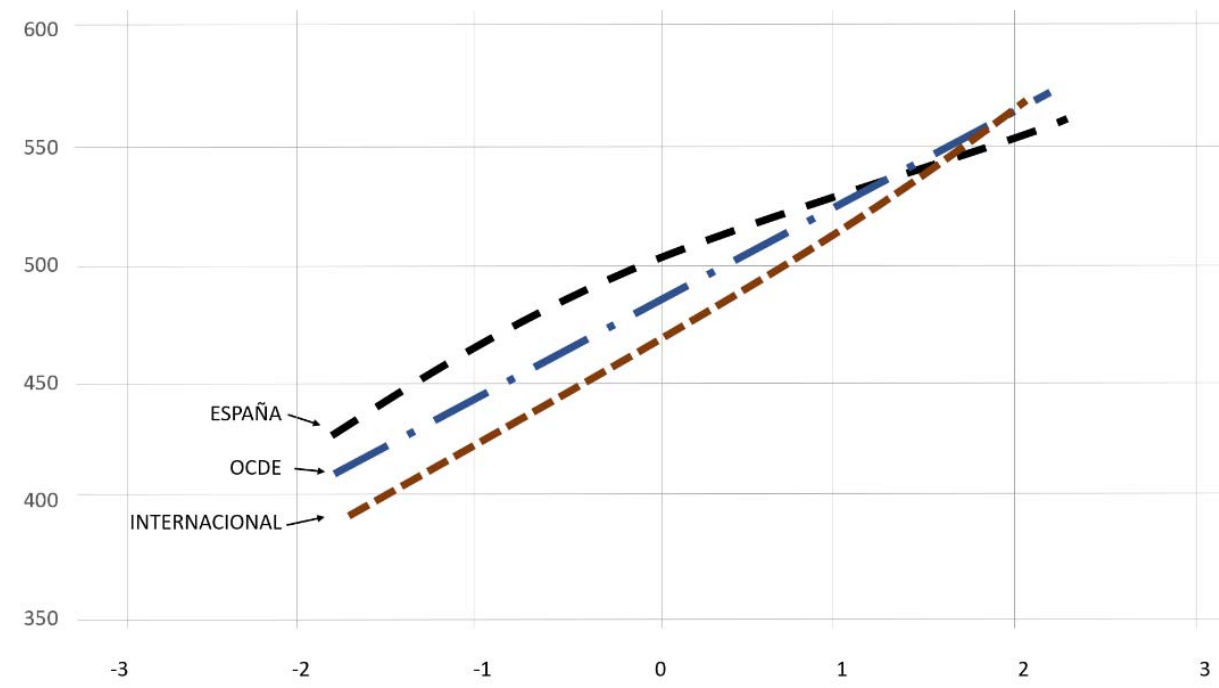

Gráfico 1. Comparación de los resultados en Lectura de España. Fuente: Ministerio de Educación, Cultura y Deporte y OCDE

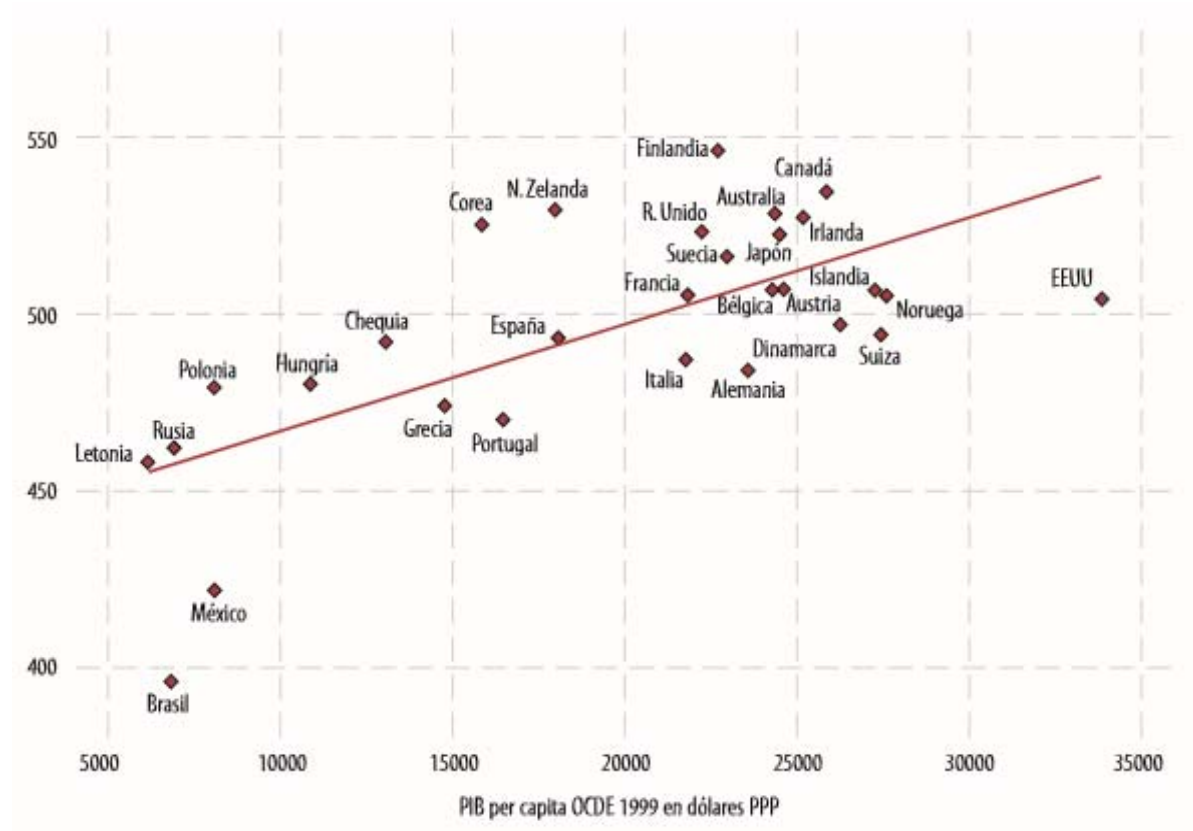

Gráfico 2. Resultados de Lectura de PISA del año 2000 y PIB. Fuente: Ministerio de Educación, Cultura y Deporte y OCDE

Cuando se analizan los resultados generales de PISA en relación al Producto Interior Bruto (PIB) de los diferentes países, se observa que España se encuentra en una tendencia central respecto a sus resultados. Nuestro país se encuentra en el grupo formado por Grecia, Portugal y España, con un PIB algo inferior que la mayoría de los 
países de su entorno, pero con unos resultados PISA que están por encima de lo esperado según su grupo de referencia (Gráfico 2).

En relación con las diferencias observadas por sexo, las alumnas mostraron un rendimiento superior (505 puntos) que los alumnos (481 puntos). Esta constatación está en consonancia con lo observado en el resto de los países participantes.

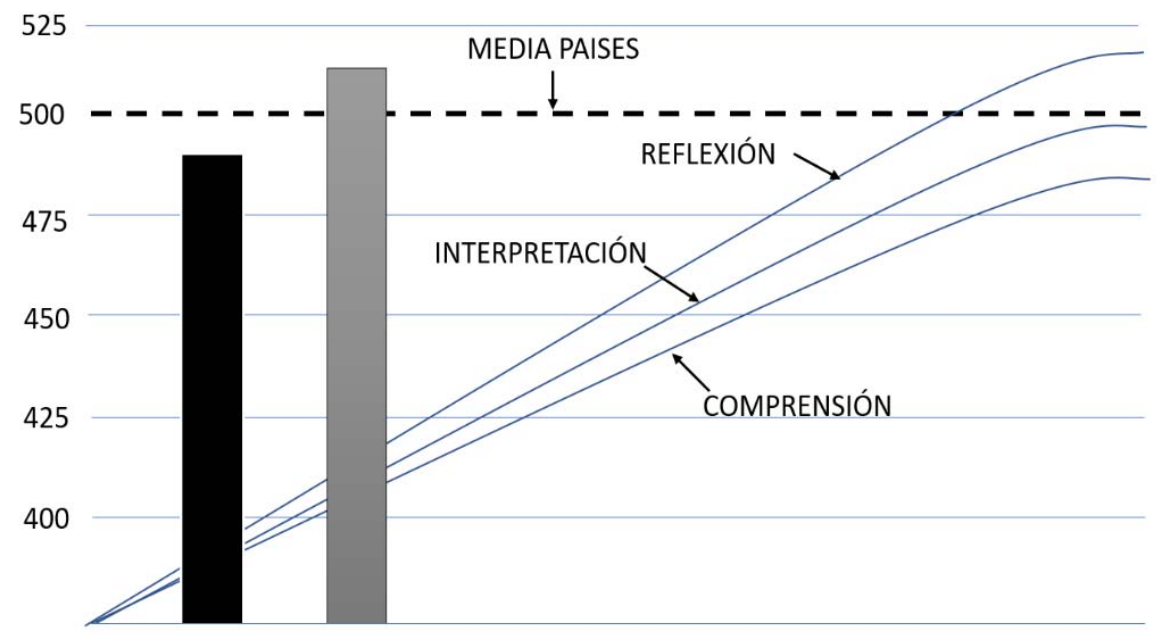

ALUMNOS ALUMNAS

Gráfico 3. Proyección de la comprensión lectora PISA 2000 y diferencias por sexo. Fuente: Ministerio de Educación, Cultura y Deporte y OCDE.

En el Gráfico 3 puede observarse cómo la puntuación media de las alumnas se sitúa por encima de la media de los países de la OCDE, mientras que la puntuación media de los alumnos se sitúa por debajo. Todo el alumnado muestra una mejor tendencia y mejores resultados en "reflexión", en comparación con "interpretación" y “comprensión". Es significativo que la variable "comprensión" se distancie tanto de la variable "reflexión", pues la primera variable es necesaria para poder obtener unos buenos resultados de la segunda.

Por otra parte, España mejora la desviación típica del conjunto de países de la OCDE (100) en 9 puntos, lo que vendría a indicar equidad e igualdad en las oportunidades ofrecidas al alumnado español, según las directrices principales del EIGE (Instituto Europeo de la Igualdad de Género). Las diferencias observadas en el alumnado derivarían sobre todo de las características familiares y personales del mismo, pero no del centro en el que el alumnado estudia.

\section{PISA 2003}

En la edición de 2003 participaron 41 países, y en España se analizaron por separado las competencias de Castilla y León, País Vasco y Cataluña. La materia principal fue Matemáticas. España, con 481 puntos, ocupa en esta edición el puesto 26 en Lectura. La nota media fue significativamente inferior a la de la edición previa (493 puntos) y se aleja de la media de la OCDE (494 puntos). En comparación con otros países, España ofrece un resultado bajo pero recupera la tendencia de mejora respecto a los informes anteriores. Finlandia tiene unos resultados un 26\% superiores a los de España. Mientras 
que Grecia ofrece un resultado un $11 \%$ inferior al de España y otros países como México incluso un 36\% por debajo de España. Las Comunidades Autónomas que ampliaron su muestra quedan por encima de la media española, aunque no siempre de un modo significativo, en competencia lectora. Castilla y León obtiene 499 puntos, País Vasco 497 y Cataluña 483 puntos (Gráfico 4).

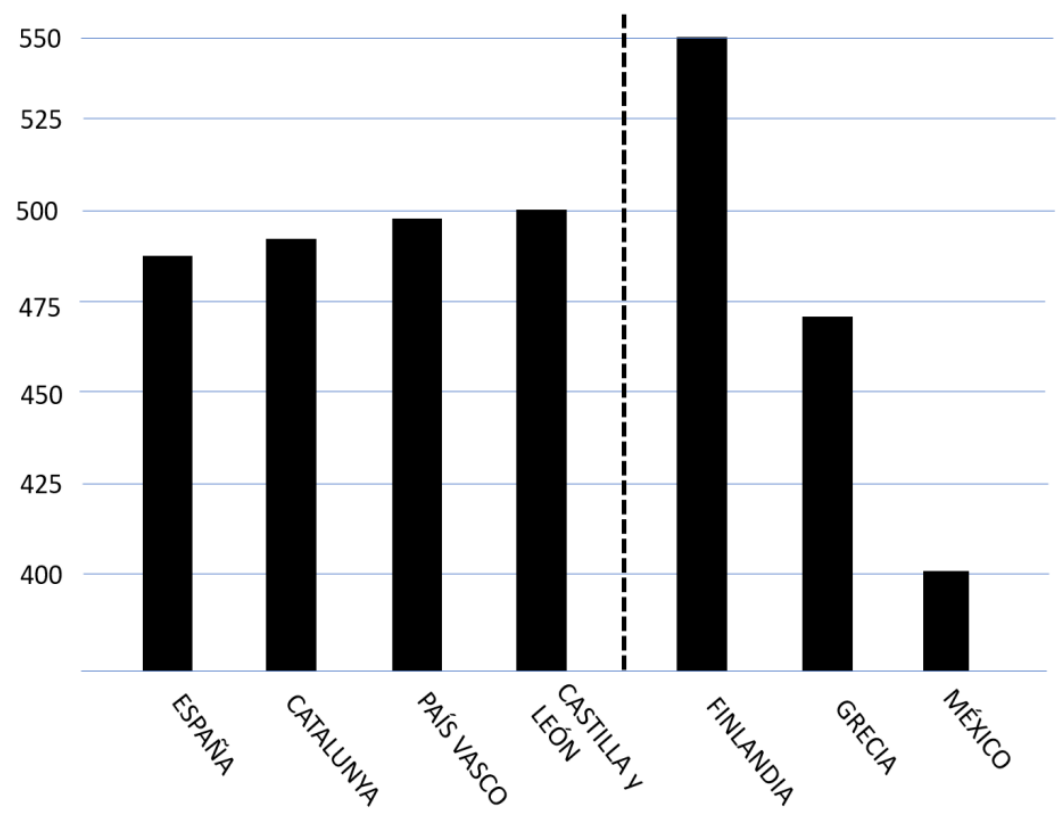

Gráfico 4. Resultados de Lectura PISA 2003. Fuente: Ministerio de Educación, Cultura y Deporte y OCDE

De nuevo, se observan diferencias entre sexos y en el mismo sentido que la edición anterior. Las alumnas presentan un rendimiento superior (500 puntos) que los alumnos (461 puntos), y la diferencia es especialmente relevante en el País Vasco. En todos los países participantes, las alumnas resultan ser mejores lectoras.

Por otra parte, España sigue posicionándose bien respecto a la equidad de los resultados, tal y como muestra la escasa dispersión de los datos.

\section{PISA 2006}

En la edición de 2006 participaron 57 países. En España se analizaron por separado las competencias de Castilla y León, País Vasco, Cataluña, Galicia, Asturias, Cantabria, La Rioja, Aragón, Andalucía y Navarra. La competencia principal evaluada fue Ciencias. En esta edición se observó un descenso en los resultados de manera generalizada en los diferentes países participantes, y en el caso español el empeoramiento fue especialmente acusado (31 puntos por debajo del promedio de la OCDE). La competencia lectora en España obtiene un promedio de 461 puntos. El resultado es también bajo en el conjunto de las Comunidades Autónomas analizadas, La Rioja obtiene 492 puntos, el País Vasco 487 puntos, Aragón 483 puntos, Navarra 481 puntos, Galicia 479 puntos, Castilla y León 478 puntos, Asturias y Cataluña 477 puntos, Cantabria 475 puntos y Andalucía 445 puntos. Tan solo La Rioja logra igualar el promedio de la OCDE (492 puntos), también se aproxima el País Vasco (487). El resto 
de las Comunidades Autónomas analizadas, salvo Andalucía, se sitúan en torno al total de la OCDE (484). Estas puntuaciones contrastan con los resultados obtenidos en Ciencias y Matemáticas en la mayoría de Comunidades Autónomas. En estas materias, el alumnado español obtiene resultados claramente superiores al promedio de la OCDE. El resto de Comunidades Autónomas analizadas se hallan cercana a la media estatal. Volviendo a la comprensión lectora, cabe subrayar el caso del País Vasco y La Rioja, Comunidades que, con buenas políticas educativas en todos los niveles educativos, logran unas puntuaciones destacables. Por el lado contrario, Andalucía llama a la atención por sus bajos resultados (Gráfico 5).

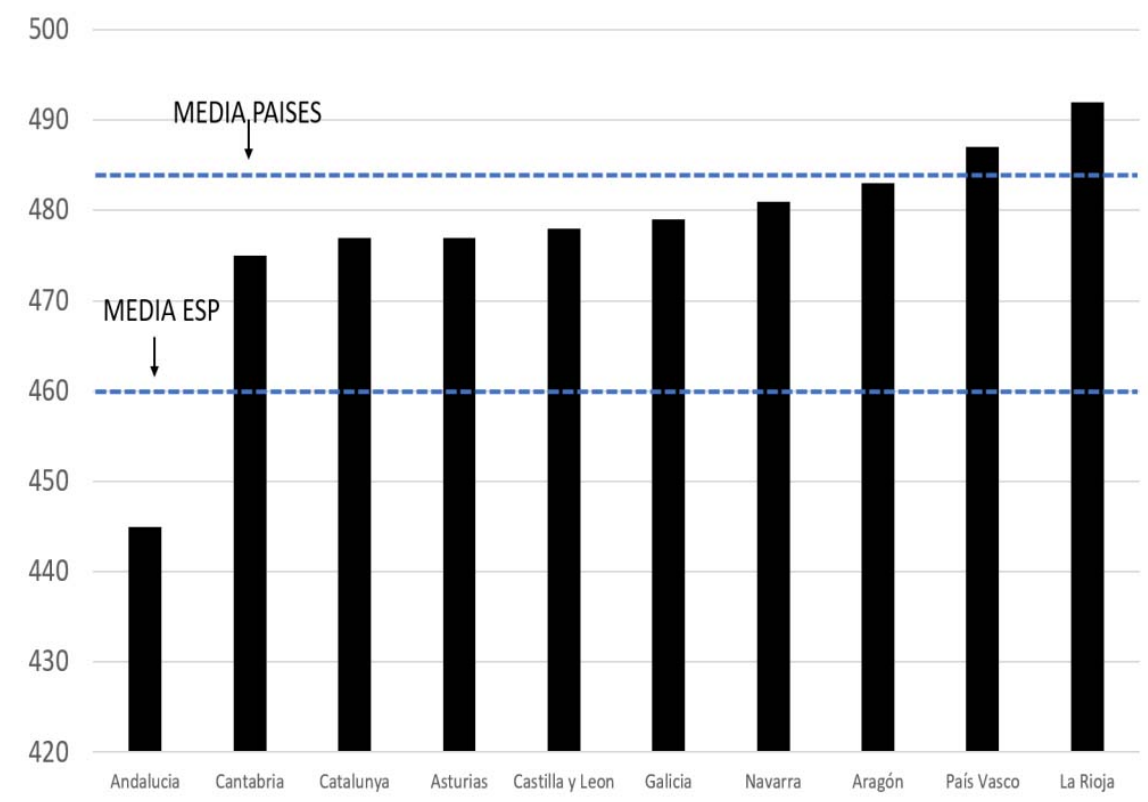

Gráfico 5. Resultados de Lectura por CC.AA. PISA 2006. Fuente: Ministerio de Educación, Cultura y Deporte y OCDE

Tal y como ocurre en el resto de los países evaluados, los resultados de las alumnas en Lectura siguen siendo superiores en España (diferencia de 35 puntos) y en todas las diferentes Comunidades Autónomas analizadas. La diferencia, en el caso español, es muy similar a la diferencia promedio en el conjunto de los países de la OCDE (38 puntos). Respecto a las Comunidades Autónomas, las diferencias resultan más importantes en Aragón y Galicia, por encima de los 40 puntos. En cambio, en Cataluña, Andalucía y Castilla y León, las diferencias a favor de las alumnas se sitúan en torno a los 25 puntos, a casi 10 puntos de la media española.

La equidad en los resultados volvería a mostrarse como un punto fuerte del sistema educativo español, se trata de un nivel próximo al de los países nórdicos.

\section{PISA 2009}

En su edición de 2009, PISA vuelve a centrarse en comprensión lectora, tal y como hizo en el año 2000. En esta edición, se incorpora la lectura de textos electrónicos y se analizan el compromiso por la lectura, el interés por la lectura y la metacognición como constructos. En 2009 se analizaron 65 países y en España se analizaron las 
competencias, por separado, de Castilla y León, País Vasco, Cataluña, Galicia, Asturias, Cantabria, La Rioja, Aragón, Andalucía, Navarra, Baleares, Canarias, Madrid, Murcia y las ciudades autónomas de Ceuta y Melilla. España obtiene un promedio de 481 puntos, mejorando la media de la edición anterior (20 puntos más) y situándose de nuevo en el resultado obtenido en la edición del 2003. El porcentaje de alumnado en los niveles más bajos de rendimiento se sitúa en el 19\%, en el promedio de la OCDE. Madrid, Castilla y León y Cataluña tienen un 13\% del alumnado en esos niveles, cifra similar a la de Japón. Entre el 14\% y el 17\% se encuentran Navarra, País Vasco, Aragón y La Rioja, además de los Países Bajos. En el 18\% se sitúan Cantabria, Asturias y Galicia, por un lado, y Suecia, Estados Unidos, Portugal, Reino Unido y Alemania, por otro. Murcia, con un $19 \%$, se sitúa cerca del promedio de la OCDE. Por encima del $25 \%$ se hallan Andalucía, Baleares y Canarias. Por otro lado, el promedio de la OCDE en los niveles altos de rendimiento es un 6\%. Castilla y León, La Rioja y Madrid poseen un $6 \%$ del alumnado en dicha franja, el 5\% del alumnado se sitúa en estos niveles en Aragón, Asturias y Navarra, el $4 \%$ del alumnado se sitúa en estos niveles en Cantabria, Cataluña y País Vasco, y en el 3\% se sitúa, junto al promedio español, Galicia. El resto de las Comunidades Autónomas presenta un 2\% del alumnado en esta franja de rendimiento.

Los resultados de las alumnas (496 puntos) siguen siendo mejor que los de los alumnos (467 puntos), tal y como sucede en las diferentes Comunidades Autónomas y en el resto de los países analizados. La diferencia, en el caso español, no es tan abultada como en otros países, la diferencia promedio en el conjunto de los países de la OCDE es de 39 puntos, y en el caso español, la diferencia es de 29 puntos. En relación con las Comunidades Autónomas analizadas, las diferencias oscilan entre los 17 puntos en Murcia y los 36 puntos en Madrid, Navarra y La Rioja. De todos modos, las diferencias en lectura a favor de las alumnas son, como puede comprobarse, de menor magnitud que el promedio de la OCDE.

Los resultados volverían a avalar la homogeneidad del sistema educativo español. La variación entre centros es del 20\%, la segunda menor tras Finlandia.

\section{PISA 2012}

En PISA 2012 participaron 65 países y en España se analizaron por separado los resultados de Andalucía, Aragón, Principado de Asturias, Illes Balears, Cantabria, Castilla y León, Cataluña, Extremadura, Galicia, La Rioja, Madrid, Región de Murcia, C. Foral de Navarra y País Vasco. Se evalúa como competencia principal el rendimiento matemático. La puntuación media del alumnado español en comprensión lectora es de 488 puntos, resultado comparable a la media de la UE (489 puntos), pero 8 puntos inferior a la media de los países de la OCDE (496 puntos), lo que sitúa a España en el puesto 23 de los 34 países de la OCDE. Asturias, Madrid, Navarra, Castilla y León, Cataluña, Galicia y País Vasco cuentan con puntuaciones medias que superan el promedio de la OCDE. Extremadura, La Rioja, Región de Murcia e Illes Balears, Cantabria y Andalucía obtienen resultados inferiores a la media de la OCDE en comprensión lectora.

Se sigue confirmando la tendencia observada en las ediciones anteriores, existe también en esta edición predominio de las alumnas (503 puntos) respecto a los alumnos en competencia lectora (474 puntos). La diferencia entre alumnas y alumnos vuelve a situarse en 29 puntos, 9 puntos menor que el promedio de la OCDE. En Castilla y León, 
las alumnas logran de media 15 puntos más en comprensión lectora que los alumnos, mientras que en Asturias esta diferencia llega a los 46 puntos. En tres Comunidades Autónomas la diferencia es mayor que el promedio de la OCDE, en dos es parecido y en el resto de las Comunidades Autónomas, la diferencia es más pequeña que en la mayoría de los países de la OCDE.

Respecto a la equidad, cabe destacar que la mayor parte de la variabilidad de los resultados se asocia a las características individuales del alumnado y no a las características de los diferentes centros, lo que apunta a que el sistema educativo español sigue siendo equitativo, a niveles comparables a los de los países nórdicos, tal y como se había constatado anteriormente.

\section{PISA 2015}

En la edición de 2015 participaron 72 países. En España se analizaron por separado la competencia matemática, lectora y científica de todas las comunidades autónomas. La competencia principal vuelve a ser la científica. En el caso español, la media en comprensión lectora se sitúa en 495 puntos, lo que nos sitúa dos puntos por encima de la media de la OCDE (493 puntos). En relación con el desempeño de las diferentes Comunidades Autónomas, las puntuaciones más elevadas en comprensión lectora corresponden a Castilla y León (522 puntos), Comunidad de Madrid (520 puntos), Comunidad Foral de Navarra (514 puntos) y Galicia (509 puntos), y sus resultados son significativamente superiores al promedio de la OCDE (493 puntos).

La diferencia según el sexo en los resultados en lectura es inferior al promedio de la OCDE, al obtener las alumnas una media de 505 puntos y los alumnos una media de 485 puntos. Esta diferencia de 20 puntos, a favor de las alumnas, es 7 puntos menor que el promedio de la OCDE. En todos los países analizados el rendimiento de las alumnas es significativamente superior al de los alumnos. Las diferencias más altas, siempre con predominio de las alumnas, se dan en Extremadura (25 puntos) y en Cantabria y Castilla y León (23 puntos) y las más bajas, en la Comunidad de Madrid (13 puntos) y la Comunidad Foral de Navarra (14 puntos). Hay que señalar que las estas últimas presentan, del mismo modo, buenos resultados en el área de lectura.

El sistema educativo español presenta, de nuevo, diferencias de las más bajas de todos los sistemas, mostrando uno de los mayores niveles de equidad entre los diferentes países y sistemas educativos analizados por el estudio.

\section{PISA 2018}

A fecha de redacción de este artículo, los datos concretos sobre comprensión lectora no habían sido publicados aún, a diferencia de los resultados sobre competencia matemática y competencia científica. 


\subsection{Resultados PIRLS (Progress in International Reading Literacy Study)}

\begin{tabular}{lccc}
\hline PIRLS & 2006 & 2011 & 2016 \\
\hline España (media) & 513 & 513 & 528 \\
\hline Alumnas & 515 & 515 & 532 \\
\hline Alumnos & 511 & 511 & 524 \\
\hline $\begin{array}{l}\text { Diferencia neta entre alumnas y alumnos (en } \\
\text { puntos) }\end{array}$ & 4 & 4 & 8 \\
\hline
\end{tabular}

Tabla 2. Media de resultados de PIRLS en España y por sexo. La diferencia observada en las diferentes ediciones resulta significativa $(\mathrm{p}=0,005)$. Fuente: Ministerio de Educación, Cultura y Deporte y la IEA (Asociación Internacional para la Evaluación del Logro Educativo)

\section{PIRLS 2006}

Se trata de la primera participación de España en esta prueba internacional. En total, participaron 40 países, la mayoría de ellos europeos. En España no se analizaron por separado las competencias de las Comunidades Autónomas. La puntuación media, obtenida por el alumnado, es de 513 puntos. Esta se sitúa en un nivel ligeramente superior al promedio internacional, pero sitúa al alumnado español lejos de las mejores puntuaciones europeas. De hecho, la mayor parte de los países europeos participantes obtienen una mejor puntuación que España.

Las alumnas presentan un mejor rendimiento en comprensión lectora (515 puntos) que los alumnos (511 puntos). España es el segundo país con menos diferencia entre alumnos y alumnas: sólo de 4 puntos, 13 puntos menos que el promedio internacional.

Según PIRLS en su edición del año 2006, el sistema educativo español no posee resultados de equidad equiparables a los de los países más equitativos de Europa, pero se muestra, a su vez, lejos de los países con sistemas escolares menos equitativos.

\section{PIRLS 2011}

En la edición de 2011 se analiza el rendimiento lector de un total de 48 países. En España fueron evaluados 8.580 alumnos de 312 centros, la muestra internacional alcanzó los 255.000 estudiantes. En esta ocasión, el informe proporciona datos complementarios de Andalucía y Canarias. En su conjunto, España obtiene 513 puntos (la misma puntuación que en la edición del 2006). Los 25 países de la OCDE alcanzan 538 puntos en promedio mientras que los de la UE logran 534. Andalucía obtiene 515 puntos y Canarias 505, en ninguno de los dos casos la diferencia es significativa.

Las alumnas españolas obtienen, en promedio, 515 puntos de puntuación, en el caso de los alumnos el promedio se sitúa en 511. La diferencia entre sexos (en rendimiento lector) es la misma que en la edición anterior, en la que la diferencia de puntuación se estableció en 4 puntos.

La edición de PIRLS 2011 confirma que España es uno de los países en donde menos influye el nivel socioeconómico de la familia en el rendimiento académico de los hijos. Además, es uno de los países que cuenta con menor diferencia entre centros. 


\section{PIRLS 2016}

En este caso, los datos españoles se completaron con el análisis de las siguientes comunidades autónomas: Andalucía, Asturias, Castilla y León, Cataluña, La Rioja, Madrid y País Vasco. El alumnado español obtiene 528 puntos en comprensión lectora, por encima del promedio PIRLS (500 puntos). El alumnado español muestra en esta edición una mejora significativa de 15 puntos en la escala de rendimiento (de 513 a 528 puntos). España, sin embargo, sigue por debajo del promedio de los países OCDE-24 (540 puntos) y del Total UE (539 puntos). En relación con el análisis por Comunidades Autónomas, Madrid (549 puntos), Asturias (548 puntos), La Rioja (546 puntos) y Castilla y León (546 puntos) presentan resultados significativamente superiores a la media del alumnado español, al promedio OCDE-24 y al total de los países de la UE. Por otra parte, las puntuaciones medias de Andalucía (525 puntos) y Cataluña (522 puntos) son inferiores, aunque no son significativamente diferentes de la puntuación media de España. En cambio, el rendimiento medio del alumnado del País Vasco (517 puntos) es significativamente menor que el promedio de España.

Con referencia a las diferencias por sexo, el rendimiento de las alumnas es mayor, significativamente, tanto en España como en el resto de los países y regiones analizados, con excepción de Portugal. Las medias de las alumnas y alumnos españoles son 532 puntos y 524 puntos respectivamente. En cuanto al análisis de las Comunidades Autónomas que amplían muestra, el rendimiento de las alumnas ha sido mejor en todas ellas, aunque la diferencia solo ha resultado significativa en el caso de Castilla y León (14 puntos), Asturias (11 puntos) y Madrid (7 puntos).

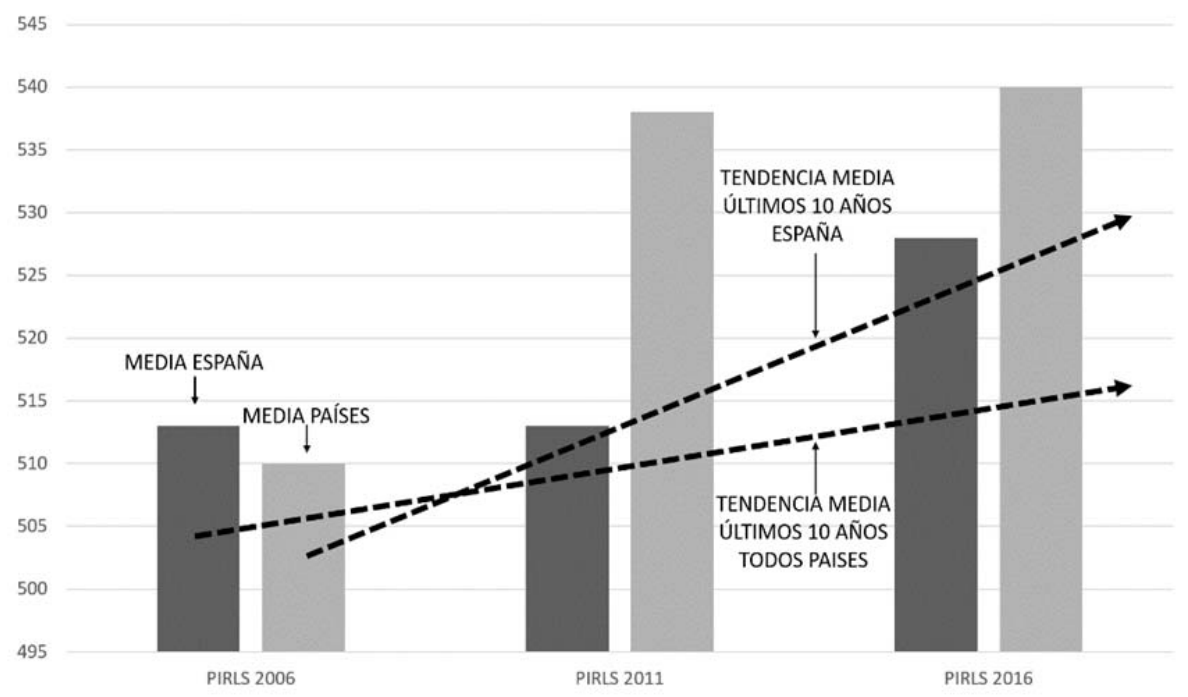

Gráfico 6. Evolución y tendencia de Lectura. PIRLS (2006-2016). Fuente: Ministerio de Educación, Cultura y Deporte y la IEA (Asociación Internacional para la Evaluación del Logro Educativo)

A partir de los resultados de los tres estudios PIRLS a nuestra disposición, puede observarse que la tendencia global de los resultados de España es al alza, a pesar de que la tendencia presenta una magnitud menor que la media de los países de los que disponemos datos. La media del resto de países muestra una tendencia que marca una diferencia del 8\% superior a la media de España (Gráfico 6). 


\subsection{Comparación brecha de género PISA y PIRLS}

El análisis conjunto de los datos en función del sexo pone en evidencia que las alumnas, de forma sostenida a través de las diferentes ediciones PISA y PIRLS, obtienen mejores resultados en comprensión lectora que los alumnos. Sin embargo, se observa una cierta diferencia entre pruebas en las tendencias constatadas (Tabla 1 y Tabla 2).

Así, según los resultados aportados por PISA, la brecha de género se ha reducido en magnitud (- 8 puntos) en la edición PISA 2015 (últimos datos disponibles) respecto a la edición previa. Tras un repunte en la edición del 2003 y el 2006, ediciones en las que la magnitud de la brecha de género fue más abultada, en PISA 2015 se reduce la diferencia en el rendimiento entre alumnas y alumnos, hasta llegar a los 21 puntos, dato que se asemeja a los 24 puntos observados en la primera edición y que supone la diferencia más pequeña en rendimiento lector, entre sexos, desde el inicio de la prueba.

Siguiendo con PISA, puede observarse, además, que la tendencia al alza del alumnado español es más pronunciada en el caso masculino, se trata de una tendencia con una diferencia porcentual del 3\% superior a la femenina. Es decir, los alumnos presentan una progresión al alza mayor que las alumnas en comprensión lectora. Este hecho lleva a pensar que, si continuase la misma tendencia, las diferencias entre alumnos y alumnas tenderán a disminuir en el futuro (Gráfico 7).

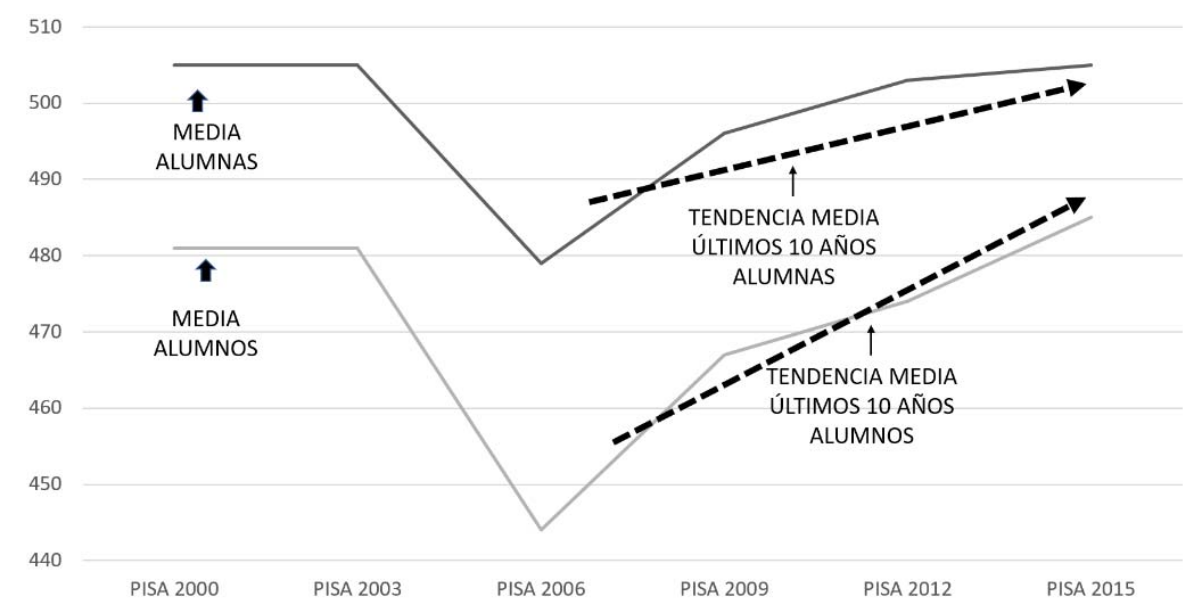

Gráfico 7. Evolución de Lectura por sexo. PISA 2000-2015. Fuente: Ministerio de Educación, Cultura y Deporte y OCDE

En cambio, y en relación con los datos proporcionados por PIRLS, la brecha de género se ha visto acrecentada, respecto a la primera y segunda edición, en 4 puntos de diferencia, siempre en beneficio de las alumnas. Las dos primeras ediciones en las que España participó (años 2006 y 2011) dejaron constancia de una diferencia entre alumnas y alumnos de 4 puntos, menor que la observada en la última edición ( 8 puntos de diferencia).

Según los datos observados en PIRLS, la tendencia de los resultados medios de las alumnas tiende a aumentar progresivamente en mayor magnitud que la tendencia de aumento progresivo de los alumnos (Gráfico 8). Se trata de un fenómeno inverso al 
constatado, y ya comentado, en PISA. No se trata de grandes diferencias entre las dos tendencias, pero muestra resultados en cierto grado diferentes a los de PIRLS.

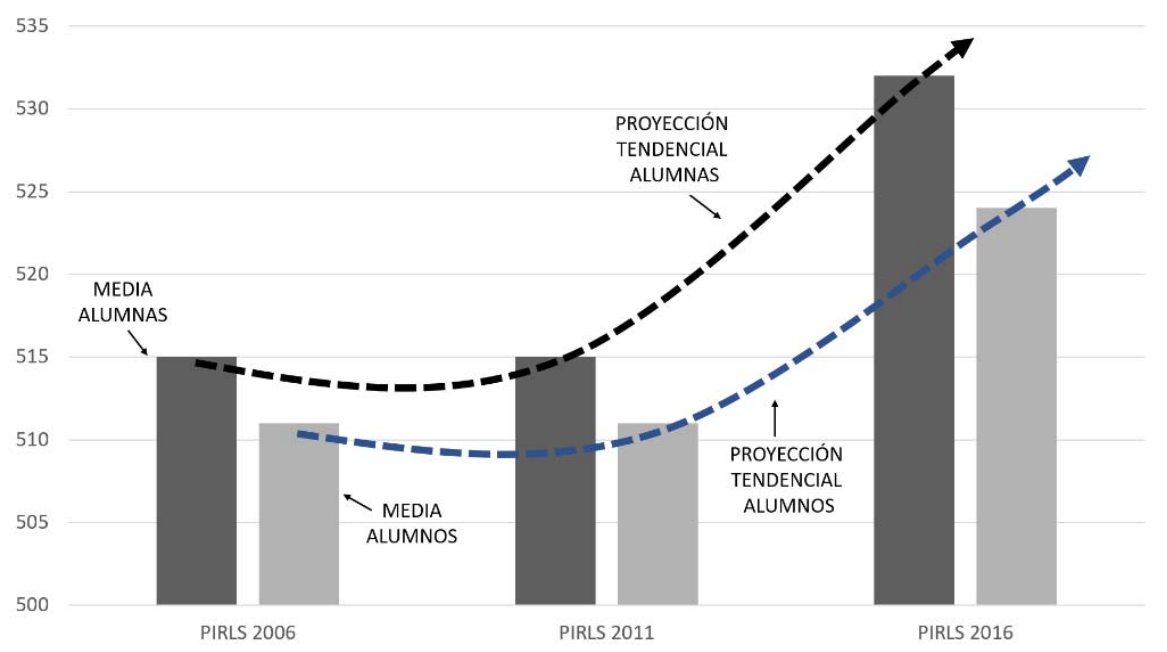

Grafico 8. Evolución y tendencias PIRLS España (2006-2016). Fuente: Ministerio de Educación, Cultura y Deporte y la IEA (Asociación Internacional para la Evaluación del Logro Educativo)

Cabe destacar que las diferencias entre sexos halladas en PIRLS, en comparación con las halladas en PISA, resultan menos abultadas (6 puntos de media en las 3 ediciones en comparación a los 29,5 puntos de media en PISA, en 6 ediciones). A pesar de que los resultados no sean equiparables al tratarse de dos pruebas distintas (metodologías y edades de los sujetos diferentes), es un dato que podría ser potencialmente interesante.

\section{CONCLUSIONES}

El análisis de las ediciones PISA y PIRLS, en las que España participó, demuestra la existencia de una brecha de género en comprensión lectora en España. Las alumnas españolas presentan mejores puntuaciones que los alumnos españoles en porcentajes que varían en función de la edición. El análisis efectuado por Comunidades Autónomas revela que dicha tendencia se repite en todas ellas a lo largo de las diferentes ediciones.

Las dos pruebas se realizan en edades diferentes, PISA en alumnado de 15 años y PIRLS en alumnado de 10 años, lo que demostraría que se trata de un fenómeno que se mantiene a lo largo del final de la infancia y principio de la adolescencia.

Hay que destacar que, según los resultados en las diferentes ediciones PISA, la brecha de género se habría reducido en la última edición (2015), algo no corroborado por la última edición PIRLS (2016). Quedaría por valorar, a través del análisis de las futuras ediciones PISA y PIRLS, cuál es la tendencia predominante a corto y medio plazo. Del mismo modo, el análisis de las futuras ediciones corroborará si la mejora de los datos en comprensión lectora globales va aparejado a una disminución en la magnitud de la brecha de género, o viceversa. Cabe subrayar que se trata de un fenómeno observado en PISA que no ha sido replicado por PIRLS.

Cara a investigaciones futuras, resultaría muy interesante también valorar las razones por las que PISA y PIRLS arrojan datos distintos en lo que se refiere a la magnitud de la brecha de género y a las tendencias en la evolución de la misma. La edad de realización 
de las pruebas (PISA 15 años) y (PIRLS 10 años) podría significar un dato que contribuya a esclarecer la cuestión.

Por otra parte, los datos más actuales revelan una mejora en comprensión lectora en el alumnado español (sin distinción de sexo). Dicha mejora se ve refrendada tanto por PISA como por PIRLS. Cabe destacar, como contrapunto, la heterogeneidad de los datos entre Comunidades Autónomas.

Los elementos expuestos en el presente trabajo permiten poner el acento en los factores contextuales para explicar las diferencias de sexo en rendimiento en comprensión lectora. Los efectos derivados del ambiente social y cultural contribuirían a la explicación de las diferencias descritas entre alumnos y alumnas.

La igualdad entre los dos sexos se muestra como un valor muy deseable en todo sistema educativo, para alcanzar esa equidad serán necesarios cambios sociales importantes y hacer efectivas políticas adecuadas. Un sistema educativo es equitativo cuando el rendimiento de sus estudiantes depende de su potencial y no de circunstancias definidas por su contexto social, económico y cultural (Sicilia y Simancas, 2018).

En los últimos años, las campañas para favorecer los estudios científicos y matemáticos para las alumnas han aumentado, habiéndose incrementado también la cuantía económica destinada a dicho efecto (González de San Román y Rica, 2012). Debería iniciarse el mismo tipo de acción con el objeto de limar diferencias entre sexos en comprensión lectora. Tal y como se ha comentado, poseer un buen nivel de comprensión lectora resulta imprescindible para que el individuo pueda manejarse en sociedad.

Del mismo modo, la mejora de la comprensión lectora del alumnado español debería convertirse en un objetivo del conjunto de la sociedad, en el que se impliquen, además de las autoridades y los agentes educativos, las familias, las instituciones y los medios de comunicación.

\section{BIBLIOGRAFÍA}

American Psychiatric Association (2000). Diagnostic and statistical manual of mental disorders (4th ed., rev.). Washington DC: American psychiatric press.

Asgarabadi, Y., Rouhi, A. y Jafarigohar, M. (2015). Learners Gender, Reading Comprehension, and Reading Strategies. Descriptive and Narrative Macro-genres. Theory and Practice in Language Studies 5. doi: 10.17507/tpls.0512.17

Bae, Y., Choy, S., Geddes, C., Sable, J. y Snyder, T. (2000). Trends in Educational Equity of Girls \& Women. Washington, DC: National Center for Education Statistics (ED). Recuperado de: https://files.eric.ed.gov/fulltext/ED440210.pdf

Blázquez-Garcés, J., Fernandez, I., Sanz, P., Tijeras, A., Vélez, X. y Pastor, G. (2015). Comprensión lectora y oral: Relaciones con CI, género y rendimiento académico de estudiantes de educación primaria. International Journal of Developmental and Educational Psychology, 1, 307-314.

Bohórquez, L.F., Cabal, M.A., y Quijano, M.C. (2014). Verbal Comprehension and Reading in Children with Reading Delay. Pensamiento Psicológico 12(1), 169-182. doi: 10.11144/Javerianacali.PPSI12-1.cvln 
Cooley, S. (1995). Suspension/Expulsion of Regular and Special Education Students in Kansas: A Report to the Kansas State Board of Education. Topeka, Kansas: Kansas State Board of Education.

Corpas Arellano, M.D. (2013). Gender differences in reading comprehension achievement in English as a foreign language in Compulsory Secondary Education. Tejuelo, 17, 67-84.

Duckworth, A.L. y Seligman, M.E.P. (2006). Self-discipline gives girls the edge: Gender in selfdiscipline, grades, and achievement test scores. Journal of Educational Psychology, 98(1), 198-208. doi: 10.1037/0022-0663.98.1.198

Elosúa, M.R., García-Madruga, J.A., Gómez-Veiga, I., López-Escribano, C., Pérez, E. y Orjales, I. (2012). Habilidades lectoras y rendimiento académico en $3^{\circ}$ y $6^{\circ}$ de Primaria: aspectos evolutivos y educativos. Estudios de Psicología 33 (2), 207-218. doi: $10.1174 / 021093912800676411$

Francis, B. (2000). Boys, Girls and Achievement: Addressing the Classroom Issues. London: Routledge Falmer.

Freeman, C.E. (2004). Trends in Educational Equity of Girls and WomIn: 2004. NCES 2005016, U.S. Department of Education, National Center for Education Statistics. Washington, D.C.: GPO,

Goldin, C., Katz, L.F. y Kuziemko, I. (2006). The Homecoming of American College Women: The Reversal of the College Gender Gap. Journal of Economic Perspectives, American Economic Association 20(4), 133-156. doi: 10.3386/w12139

González de San Román, A. y Rica, S. (2012). Gender Gaps in PISA Test Scores: The Impact of Social Norms and the Mother's Transmission of Role Attitudes. IZA Institute for the Study of Labor Discussion Paper Serie, 34.

Gregory, J.F. (1996). The Crime of Punishment: Racial and Gender Disparities in the Use of Corporal Punishment in the U.S. Public Schools. Journal of Negro Education, 64, 454-462. doi: $10.2307 / 2967267$

Heckman, J.J., Stixrud, J. y Urzua, S. (2006). The Effects of Cognitive and Noncognitive Abilities on Labor Market Outcomes and Social Behavior. Journal of Labor Economics 24(3), 411482. doi: $10.3386 /$ w 12006

Jacob, B.A. (2002). Where the Boys Aren't: Noncognitive Skills, Returns to School and the Gender Gap in Higher Education. Economics of Education Review 21(6), 589-598. doi: $10.3386 /$ w8964

Julià, A. (2016). Contexto escolar y desigualdad de género en el rendimiento de comprensión lectora. Revista Española de Investigaciones Sociológicas 156, 41-58. doi: $10.5477 /$ cis/reis. 156.41

Koban, D. (2016). The Role of Gender in Reading Comprehension: An Analysis of College-level EFL Students' Comprehension of Different Genres. International Online Journal of Education and Teaching (IOJET), 3, 218-227.

León, A., Amaya, S. y Orozco, D. (2012). Relación entre comprensión lectora, inteligencia y desempeño en pruebas Saber Pro en una muestra de estudiantes universitarios. Cultura educación y sociedad, 3 (1). Recuperado de:

https://revistascientificas.cuc.edu.co/culturaeducacionysociedad/article/view/964

Martín, E. (2010). La escuela sin funciones. Barcelona: Ediciones Bellaterra. 
McGeown, S., Goodwin, H., Henderson, N. y Wright, P. (2011). Gender differences in reading motivation: Does sex or gender identity provide a better account? Journal of Research in Reading 35 (3), 328-336. doi: 10.1111/j.1467-9817.2010.01481.x.

McGeown, S. (2012). Sex or gender identity? Understanding children's reading choices and motivation. Journal of Research in Reading 38(1), 35-46. doi: 10.1111/j.14679817.2012.01546.x

Ministerio de Educación y Ciencia (2007). PIRLS 2006. Estudio Internacional de Progreso en Comprensión Lectora de la IEA. Informe español. Madrid: Ministerio de Educación y Ciencia. Recuperado de: https:/www.educacionyfp.gob.es/inee/dam/jcr:01b7dbb0-99444c30-ab73-2fffaadd8703/pirls2006.pdf

Ministerio de Educación, Cultura y Deporte (2013). PIRLS - TIMS 2011. Estudio Internacional de progreso en comprensión lectora, matemáticas y ciencias. IEA. Volumen I. Informe Español. Madrid: Ministerio de Educación y Ciencia. Recuperado de: http://www.educacionyfp.gob.es/inee/dam/jcr:536b9019-936b-4eb6-a46615459c87f1a9/pirlstimss2011vol1-1.pdf

Ministerio de Educación, Cultura y Deporte (2017). PIRLS 2016 Estudio internacional de progreso en comprensión lectora. Informe español. Madrid: Ministerio de Educación y Ciencia. Recuperado de: http://www.educacionyfp.gob.es/inee/dam/jcr:64541373-26c2$4 \mathrm{e} 0 \mathrm{f}-\mathrm{b} 66 \mathrm{c}-$

79168c44bbec/PIRLS\%202016\%20INFORME\%20NACIONAL_ONLINE_20dic.pdf

Ministerio de Educación y Formación Profesional (2019). Informe PISA 2018. Madrid: Ministerio de Educación y Ciencia. Recuperado de: https://rive.google.com/file/d/1QOkG8vq1GzhAuNP7mHLtQm3iCqt8wC8G/view

Moffitt, T.E. (ed.) (2001). Sex Differences in Antisocial Behavior: Conduct Disorder, Delinquency, and Violence in the Dunedin Longitudinal Study. Cambridge: Cambridge University Press. ISBN 0521804450. Recuperado de: https://assets.cambridge.org/97805218/04455/frontmatter/9780521804455_frontmatter.pdf

Moje, E.B., Stockdill, D., Kim, K. y Kim, H.J. (2011). The Role of Text in Disciplinary Learning. En M.L. Kamil, P.D. Pearson, E.B. Moje y P.P. Afflerbach (Eds.), Handbook of Reading Research, (pp. 453-486). Nueva York: Routledge.

Mol, S.E. y Jolles, J. (2014). Reading enjoyment amongst non-leisure readers can affect achievement in secondary school. Frontiers in Psychology, 5, 1.214-1.229. doi: 10.3389/fpsyg.2014.01214

Mullis, I., Martin, M. González, E. y Kennedy, A. (2003). PIRLS 2001 International Report. IEA's Study of Reading Literacy Achievment in Primary Schools in 35 countries. International Association for the Evaluation of Educational Achievment - International Study Center, Lynch School of Education, Boston College.

OCDE (2002). Conocimientos y aptitudes para la vida. Primeros resultados del Programa Internacional de Evaluación de Estudiantes PISA (2000) de la OCDE. México: Aula XXI.

OCDE (2004). Informe PISA 2003. Aprender para el mundo del mañana. Madrid: Ediciones Santillana y Ministerio de Educación y Ciencia.

OCDE (2008). Informe PISA 2006. Competencias científicas para el mundo del mañana. Madrid: Ediciones Santillana y Ministerio de Educación y Ciencia.

OECD (2010). PISA 2009 Results: Overcoming Social Background: Equity in Learning Opportunities and Outcomes (Volume II). París: OECD Publishing.

OCDE (2010a). PISA 2009. Vol II. Overcoming Social Background. París: OECD Publishing. 
OCDE (2010b). PISA 2009. Vol. III. Learning to learn. París: OECD Publishing.

OCDE (2010c). PISA 2009: What Students Know and Can Do. Vol. I. París: OECD Publishing.

OECD. (2015). The ABC of gender equality in education: aptitude, behaviour, confidence. París: OECD Publishing.

Olivares, F., Fidalgo, R. y Torrance, M. (2016). Diferencias en la auto-eficacia lectora entre cursos en la escolaridad y en función del género. Revista de Psicodidáctica 21(1), 45-63. doi: $10.1387 /$ RevPsicodidact. 13832

Rosenbaum, J.E. (2001). Beyond College for All: Career Paths for the Forgotten Half. New York: Russell Sage.

Rosselli, M., Matute, E. y Ardila, A. (2006). Predictores neuropsicológicos de la lectura en español. Revista de Neurología 42(4), 202-21. Recuperado de: https://pdfs.semanticscholar.org/ffec/0d12dc85a1b2c209f3160c14ee473f034967.pdf

Sicilia, G. y Simancas, R. (2018). Equidad educativa en España: comparación regional a partir de PISA 2015. Madrid: Fundación Ramón Areces. Recuperado de: https://www.fundacionareces.es/recursos/doc/portal/2018/03/20/equidad-educativa-enespana.pdf

Skelton, C. (1997). Primary Boys and Hegemonic Masculinities. British Journal of Sociology of Education 18(3), 349-369. doi:10.1080/014256997018030

Skiba, R.J., Michael, R.S., Nardo, A.C. et al. (2002). The Color of Discipline: Sources of Racial and Gender Disproportionality in School Punishment. The Urban Review 34, 317-342. doi: 10.1023/A:1021320817372

Torres, P. y Granados, D.E. (2014). Procesos cognoscitivos implicados en la comprensión lectora en tercer grado de educación primaria. Psicogente 17(32), 452-459.. Recuperado de: http://www.scielo.org.co/pdf/psico/v17n32/v17n32a16.pdf

Trzesniewski K.H., Moffitt T.E., Caspi, A., Taylor, A. y Maughan, B. (2006). Revisiting the association between reading achievement and antisocial behavior: new evidence of an environmental explanation from a twin study. Child Development, 77(1), 72-88. doi:10.1111/j.1467-8624.2006.00857.x

Velarde, E., Canales, R. y Meléndez, M. (2013). Procesos psicológicos de la lectura en estudiantes de primaria del callao, según nivel socioeconómico y género. Revista de Investigación en Psicología 16(1), 153-170.

Weiner, G. (2010) Gender and education in Europe: a literature overview. En Gender Differences in Educational Outcomes: Study of the Measures Taken and the Current Situation in Europe (pp. 15-32). Bruselas: Eurydice.

Yazdanpanah, L.K.. (2007). The Effect of Background Knowledge and Reading Comprehension Test Items on Male and Female Performance. The Reading Matrix: An International Online Journal, 7(2), 64-80. 